\title{
Niveles de plomo en la población de alto riesgo y su entorno en San Ignacio, Fresnillo, Zacatecas, México
}

\author{
Eduardo Manzanares-Acuña, $\mathrm{Dr}$ en C,(1) Héctor René Vega-Carrillo, $\mathrm{PhD}$,(I) \\ Miguel Ángel Salas-Luévano, Dr en C, (I) Víctor Martín Hernández-Dávila, M en C,(I) \\ Consuelo Letechipía-de León, M en C, (') Rómulo Bañuelos-Valenzuela, Dr en C, (I)
}

\begin{abstract}
Manzanares-Acuña E,Vega-Carrillo HR, Salas-Luévano MA, Hernández-Dávila VM, Letechipía-de León C, Bañuelos-Valenzuela R. Niveles de plomo en la población de alto riesgo y su entorno en San Ignacio, Fresnillo, Zacatecas, México. Salud Publica Mex 2006;48:21 2-219.
\end{abstract}

\begin{abstract}
Resumen
Objetivo. Determinar la concentración de plomo en la sangre de niños y mujeres, en periodo de lactancia o embarazadas, de la comunidad de San Ignacio, Fresnillo, en Zacatecas, así como en matrices de suelo, plantas, ceniza y barro vidriado, para determinar la exposición que genera una empresa recicladora de metales. Material y métodos. El estudio se realizó de diciembre de 2004 al mes de abril de 2005 . El Pb en sangre se midió por voltamperometría de redisolución anódica, mientras que se utilizó energía dispersa de fluorescencia por rayos $\mathrm{X}$ para las otras matrices. Resultados. Con base en los criterios de la Norma Oficial Mexicana (NOM) se encontró que $90 \%$ de los menores está en la categoría I, $5 \%$ se encuentra en la categoría ll y el otro $5 \%$ se ubica en la categoría III. El suelo de los terrenos aledaños a la recicladora tiene en promedio $4940 \mu \mathrm{g} / \mathrm{g}$, en un intervalo de 73 a $84238 \mu \mathrm{g} / \mathrm{g}$. Las mayores concentraciones se localizan en los puntos más cercanos a la empresa. El suelo de San Ignacio tiene en promedio $109 \mu \mathrm{g} / \mathrm{g}$. Se encontraron valores altos en el barro vidriado y la concentración del $\mathrm{Pb}$ en las plantas es superior a los $300 \mu \mathrm{g} / \mathrm{g}$. Conclusiones. A pesar de que la mayoría de los niños analizados presenta niveles de plomo en sangre que la Norma Mexicana señala como aceptables, varios estudios indican que existen efectos nocivos aun a concentraciones bajas. Los terrenos aledaños a la empresa están contaminados, y en esa medida los alimentos que ahí se producen, al ser consumidos, son una fuente de contaminación, que se agudiza con el uso de barro vidriado.
\end{abstract}

Palabras clave: niño; intoxicación por plomo; sangre; suelo; México

\section{Cuerpo Académico de Radiobiología, Universidad Autónoma de Zacatecas, México.}

Fecha de recibido: II de mayo de 2005 - Fecha de aceptado: 16 de febrero de 2006

Solicitud de sobretiros: Mtro. Héctor René Vega-Carrillo. Cuerpo Académico de Radiobiología,

Universidad Autónoma de Zacatecas, Apdo. Postal 336, 98000 Zacatecas, Zac., México

Correo electrónico: rvega@uaz.edu.mx
Manzanares-Acuña E,Vega-Carrillo HR, Salas-Luévano MA, Hernández-Dávila VM, ad levels in high-risk populations and the surrounding environment in San Ignacio, Fresnillo, Zacatecas, México. Salud Publica Mex 2006;48:21 2-219.

ective. To determine the lead concentration in the blood tildren and nursing or pregnant women from San Ignash and lead-glazed pottery, in order to determine sure due to a metal-recycling facility. Material and Methods. The study was carried out from December 2004 to April 2005. Lead in blood was measured with anodic strip作 used in the other matrices. Results. Based upon the and another $5 \%$ as category III. The soil in the land near the facility contained from 73 to $84238 \mu \mathrm{g} / \mathrm{g}$, with an average of $4940 \mu \mathrm{g} / \mathrm{g}$. Larger lead concentrations were found on closer to the facility. San Ignacio's soil conpottery and the concentration in agricultural crops majority of children in San lgnacio have blood lead concen trations considered to be acceptable according to the Official Mexican Standards, several studies indicate that delerious effects on children's health exist even at low is contaming are produced there, once ingested, are a source of contamination, which is compounded by the use of glazed pottery.

Key words: child; lead poisioning; blood; soil; Mexico 
E plomo $(\mathrm{Pb})$ es un metal no esencial para los seres humanos que se distribuye con amplitud en el ambiente; se considera un contaminante ecotoxicológico ya que su uso provoca contaminación ambiental y exposición en humanos. ${ }^{1}$ Se emplea en la fabricación de pinturas, como antidetonante de la gasolina, en la fabricación de cerámica, reciclaje de baterías, manejo de residuos tóxicos industriales y es común en actividades mineras. La principal vía de biodisponibilidad son el suelo y el polvo, donde se concentra y por medio del cual ingresa al organismo por ingestión e inhalación. ${ }^{2-4} \mathrm{Su}$ biodisponibilidad es un problema de salud en niños, embarazadas y mujeres en periodo de lactancia. A este grupo de la población se le considera como el principal grupo en riesgo. En niños, una concentración de $10 \mu \mathrm{g}$ de $\mathrm{Pb}$ por decilitro de sangre ocasiona daños irreversibles en el sistema nervioso central, retraso en el crecimiento y afecta al sistema hematopoyético, hepático y endocrino. ${ }^{5,6}$ Cuando el polvo con $\mathrm{Pb}$ se deposita en los objetos y el suelo, los niños resultan ser los más expuestos por el hábito de llevarse a la boca sus manos y objetos contaminados con ese polvo. Las deficiencias nutricionales de hierro, zinc, cobre, calcio y fósforo en los infantes potencian la absorción del plomo. ${ }^{7,8}$ El uso de loza vidriada, horneada a baja temperatura, así como algunos remedios tradicionales, como la "greta" y el "azarcón", que se emplean como tratamiento para el "empacho" y la diarrea, son fuentes de contaminación por su alto contenido de plomo. El empleo de este tipo de remedios ha producido intoxicación en niños mexicanos. ${ }^{9}$

Entre los habitantes de las comunidades de San Ignacio, Río Florido, Bañuelos y la colonia Morelos, pertenecientes al municipio de Fresnillo en el estado de Zacatecas, existe inquietud por las actividades de una empresa denominada Reciclado de Metales Gildardo Gómez Alonso, que se ubica en medio de una zona agropecuaria y a una distancia aproximada de 500 metros de la comunidad de San Ignacio. Una de las actividades de la empresa es la desbismutación del plomo, donde se manejan compuestos ricos en este metal; una vez recuperado el bismuto, el resto se convierte en desecho. El manejo inadecuado de materiales con plomo ha sido causante de numerosos problemas ambientales en diversos lugares del estado de Zacatecas. ${ }^{10}$

En este estudio se midió la concentración de plomo en la sangre de niños, embarazadas y mujeres en periodo de lactancia. También, se determinó la concentración de plomo del suelo, de calles y casas de la comunidad, así como de los terrenos, agrícolas y de agostadero, ubicados en torno a la recicladora. Algunos productos de estos terrenos de cultivo también se analizaron.

\section{Material y métodos}

La comunidad de San Ignacio se ubica a $23^{\circ}, 19^{\prime}$ norte y $102^{\circ}, 59^{\prime}$ oeste. Es el centro de población más próximo a la planta recicladora. De los 253 niños menores de 15 años de San Ignacio, se determinó un tamaño de muestra de 59 bajo los siguientes supuestos: nivel de significancia $95 \%$, proporción de niños afectados $5 \%$. Por grupo de edad, la selección de niños se distribuyó de la siguiente forma: $15 \%$, de los niños seleccionados tenía entre 0 y 3 años; $15 \%$ entre 3 y 6; 50\% de los menores, entre 6 y 12 años; y 20\%, entre 12 y 15 años. Debido a que no se cuenta con información confiable sobre el total de mujeres en periodo de lactancia y embarazadas, en el estudio se decidió tomar muestras de aquellas que accedieran a participar. Los niños se identificaron por grupo de edad y los individuos se seleccionaron en forma aleatoria hasta alcanzar el tamaño de la muestra.

A cada participante se le lavaron las manos con agua y jabón; después del secado, se desinfectó el dedo anular con alcohol. La sangre se obtuvo mediante punción con lanceta del dedo anular y se recuperó con un tubo capilar con EDTA de $50 \mu \mathrm{l}$, que se mezcló con el reactivo (250 $\mu \mathrm{l}$ de ácido clorhídrico diluido al $0.1 \mathrm{~mol} / \mathrm{L}$ en agua), lo cual permite la digestión de la sangre y la liberación del plomo.

La concentración de $\mathrm{Pb}$ en sangre se determinó mediante voltamperometría de redisolución anódica mediante el sistema LEADCARE de la empresa ESA, Inc., * y el procedimiento que recomienda la Norma Oficial Mexicana. ${ }^{11}$ El equipo se calibró a partir de dos estándares con diferentes concentraciones de plomo. Este control se aplicó al inicio y al final de las mediciones y cada 10 determinaciones. El coeficiente de variación de la técnica es del 5\%, obtenido al medir un mismo estándar en ocho ocasiones. Para determinar si existe una diferencia significativa entre la concentración de plomo en niños y niñas, se aplicó la prueba no paramétrica U de Mann y Whitney ${ }^{12}$ a cada grupo de edad y al conjunto sin distinción de edades. Esta prueba permitió determinar si existían diferencias de significancia estadística entre el promedio de $\mathrm{Pb}$ en sangre de las niñas y el promedio de $\mathrm{Pb}$ en sangre de los niños.

En dos visitas a la zona se obtuvo un total de 34 muestras de suelo del área próxima a la empresa. ${ }^{13}$ En la figura 1 se indica con cuadrados los sitios del primer muestreo y con círculos oscuros los sitios del se-

* ESA, Inc. 22 Alpha Road Chelmsford, MA. EUA. 
gundo muestreo. La muestras se tomaron a nivel de la superficie, es decir a una profundidad menor a $10 \mathrm{~cm}$. En la comunidad de San Ignacio se tomaron 17 muestras de suelo, 12 son de puntos ubicados en las calles y cinco de los patios de tres casas habitación correspondientes a los tres grupos familiares, que en lo sucesivo se designan como familias 1, 2 y 3 . De estas mismas casas se tomaron muestras de las cenizas generadas de la combustión de leña, ya que se obtiene de la zona donde se encuentra la recicladora.

Las casas de donde se tomaron muestras de suelo y cenizas corresponden a familias donde al menos uno de sus miembros tuvo concentraciones de PB en san- gre superiores a los $10 \mu \mathrm{g} / \mathrm{dl}$. En las familias 2 y 3 se utiliza leña, como complemento del gas, para cocinar, mientras que en la familia 1 la leña sólo se emplea para alimentar al calentador de agua. De la familia 2 se obtuvieron muestras de nopal duraznillo (Opuntia leucotricha) que utilizan como alimento y que la familia obtuvo del área de la recicladora. La familia 3 es la única de las tres que utiliza barro vidriado para la preparación de sus alimentos. En los últimos 10 años han utilizado un par de ollas de barro vidriado para cocer frijoles (Phaseolus vulgaris), alimento que forma parte de su dieta diaria. Ambos recipientes se recuperaron, y uno de éstos se utilizó para determinar la cantidad

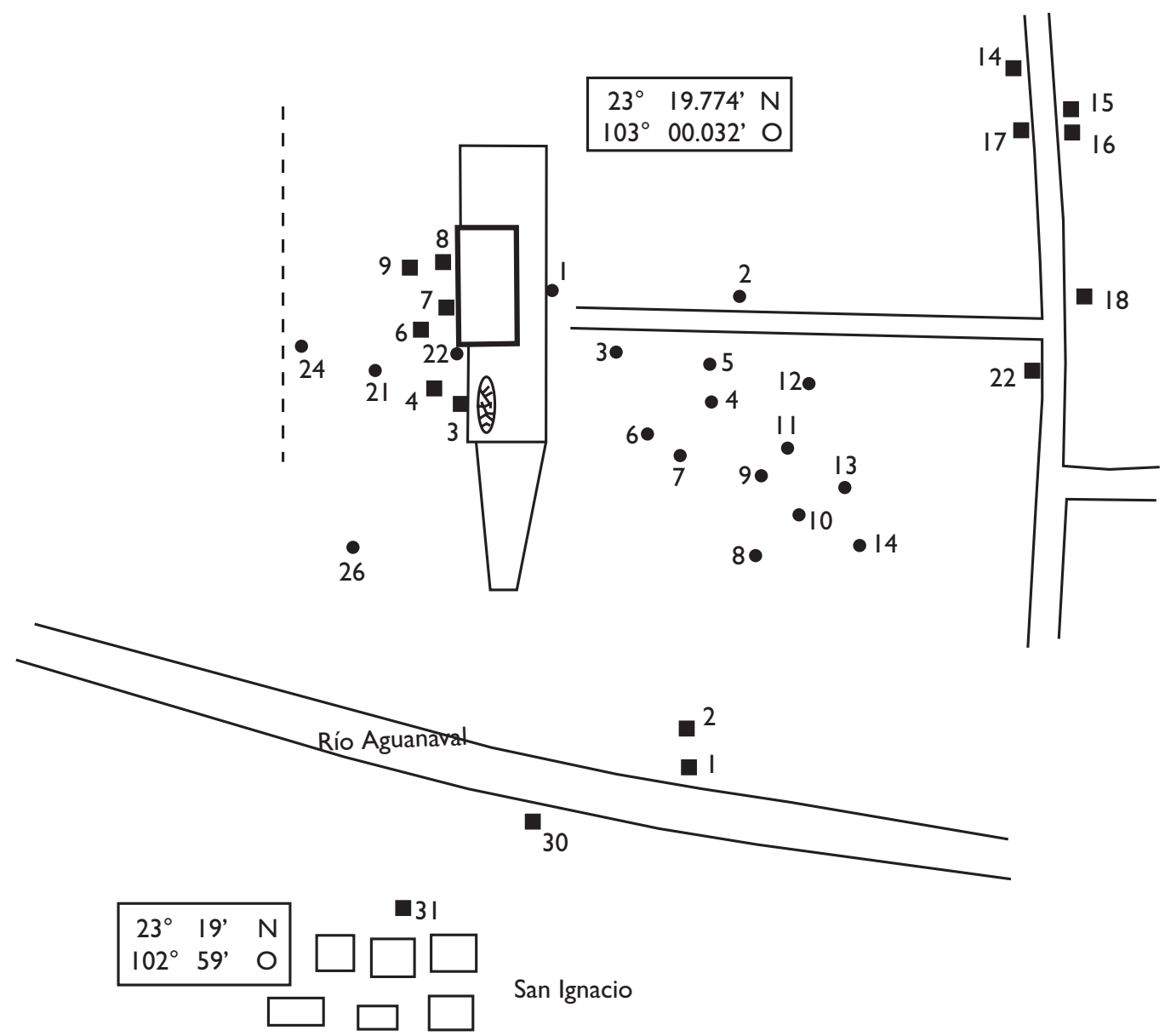

Figura I. Lugares de la comunidad de San Ignacio, Fresnillo, Zacatecas, México, donde se tomaron las MUESTRAS DE SUELO. LOS CUADRADOS CORRESPONDEN A LAS MUESTRAS DE LA PRIMERA VISITA, REALIZADA DURANTE EL MES DE ENERO DE 2005, Y LOS CíRCULOS OSCUROS, A LAS DE LA SEGUNDA VISITA, EFECTUADA DURANTE EL MES DE ABRIL DE

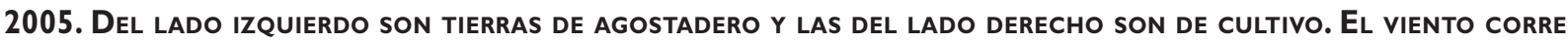
PREFERENTEMENTE DE IZQUIERDA A DERECHA 
de $\mathrm{Pb}$ que contiene, mientras que el otro se usó para medir la cantidad de $\mathrm{Pb}$ que se desprende durante la cocción de frijoles.

De los terrenos de cultivo aledaños a la empresa se obtuvieron muestras de camote (Hipomoea batata) y de chile (Capsicum annum); para establecer una base de comparación, se utilizó como testigo chile de Bañuelos, Guadalupe, que se encuentra aproximadamente a $70 \mathrm{~km}$ de distancia de la zona de estudio. Las muestras de suelo, frijoles crudos y cocidos en la olla de barro vidriado, chile, camote, nopal y las cenizas se colocaron en una estufa a $40^{\circ} \mathrm{C}$ para eliminar la humedad. Una vez secos, se molieron y tamizaron hasta obtener un polvo fino de 350 mallas. ${ }^{3}$ Una de las ollas de barro se utilizó para determinar la concentración de plomo en las partes interna y externa de la olla. Los niveles de plomo en las muestras se midieron en cinco ocasiones mediante Energía Dispersa de Fluorescencia de Rayos X (EDXRF), con un equipo de Phillips modelo MiniPal. El sistema se calibró mediante 12 estándares, cuatro de ellos pertenecientes al Instituto Nacional de Estándares y Tecnología de Estados Unidos (NIST): Montana soil 2710 y NBS 1570, 1573, 1575. La curva de calibración se determinó mediante regresión lineal por mínimos cuadrados ponderados. ${ }^{14}$ El factor de ponderación utilizado se muestra en la siguiente ecuación:

$$
\omega_{\mathrm{i}}=\frac{1}{\left(\mathrm{~S}_{\mathrm{STD}}\right)_{\mathrm{i}}+\left(\mathrm{S}_{\mathrm{CPS}}\right)_{\mathrm{i}}}
$$

donde $\omega_{\mathrm{i}}$ es el factor de ponderación del $i$-ésimo estándar, $\left(\mathrm{s}_{\mathrm{STD}}\right)_{i}$ es la varianza de la cantidad de plomo contenido en el $i$-ésimo estándar y $\left(\mathrm{s}_{\mathrm{CPS}}\right)_{\mathrm{i}}$ es la varianza del promedio de las cuentas obtenidas bajo el pico de plomo en el espectrómetro de EDXRF para el $i$-ésimo estándar.

Al colocar una muestra en el espectrómetro de EDXRF se observa el espectro de todos los elementos que contiene, desde el Al hasta el U. La posición de los picos indica el tipo de elemento, y el área bajo la curva señala la concentración. Mediante el proceso de calibración el espectrómetro selecciona la parte del espectro que corresponde al $\mathrm{Pb}$, calcula el área bajo la curva del pico y suministra como resultado la concentración de $\mathrm{Pb}$. Para verificar la sensibilidad y especificidad de la EDXRF, un conjunto de muestras se analizó por esta técnica y por la técnica de absorción atómica con horno de grafito (AA); ambos procedimientos arrojaron los mismos resultados en un intervalo entre 100 y $5000 \mu \mathrm{g} / \mathrm{g}$, como se muestra en la figura 2. Ambos procedimientos permiten determi-

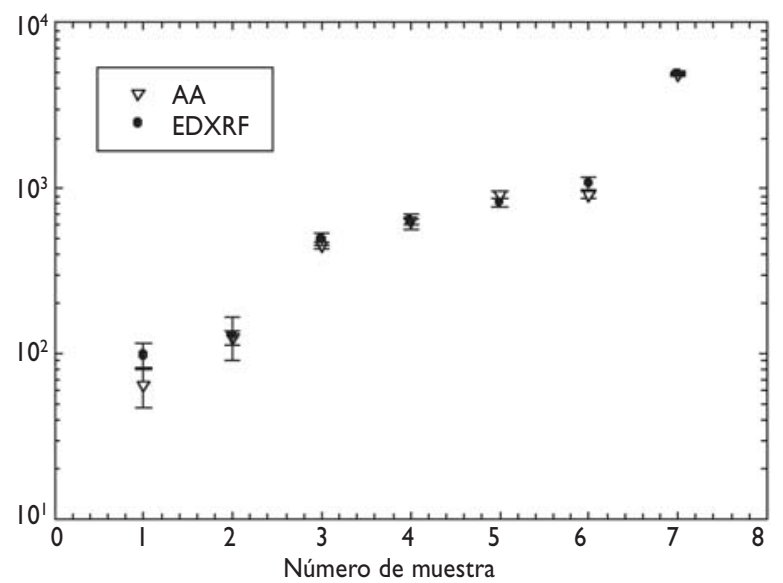

Figura 2. Concentración de Pb en siete muestras DETERMINADA POR ABSORCIÓN ATÓMICA CON HORNO DE GRAFITO (AA) Y POR ENERGÍA DISPERSA DE FLUORESCENCIA DE RAYOS X (EDXRF), SAN IGNACIO, ZaCatecas

nar concentraciones de plomo en muestras de suelo tan pequeñas como $100 \mu \mathrm{g} / \mathrm{g}$.

\section{Resultados}

De los 59 niños que participaron en el estudio, $47 \%$ son varones y $53 \%$ son mujeres. El estudio incluyó a cinco embarazadas o en periodo de lactancia. La concentración de plomo en sangre se muestra en la figura 3, que refleja los valores límites establecidos por la NOM. ${ }^{11}$ En su conjunto, todos los menores tienen en promedio una concentración de plomo en sangre de $4.9 \pm 3.6 \mu \mathrm{g} / \mathrm{dl}$, mientras que las adultas tienen $2.2 \pm 0.2 \mu \mathrm{g} / \mathrm{dl}$. En el cuadro I se muestra el valor promedio de la concentración de $\mathrm{Pb}$ en sangre por género, grupo de edad y en conjunto. El porcentaje de los menores, por género y grupo de edad así como en su conjunto, que tiene concentraciones de plomo en sangre $\geq 10 \mu \mathrm{g} / \mathrm{dl}$ se muestra en el cuadro II. En la figura 4 se presentan las concentraciones de $\mathrm{Pb}$ en el suelo de los terrenos cercanos a la recicladora en función del número de muestra asignado en la figura 2, así como las del suelo de la población.

El promedio de plomo en el suelo de los terrenos vecinos a la recicladora es $4940 \pm 14950 \mu \mathrm{g} / \mathrm{g}$, en un intervalo que varía de 73 a $84238 \mu \mathrm{g} / \mathrm{g}$. Las muestras 10 y 26 del segundo muestreo presentan concentraciones inferiores a los $200 \mu \mathrm{g} / \mathrm{g}$. La muestra 10 se obtuvo a una profundidad mayor a los $50 \mathrm{~cm}$ con el propósito de determinar la concentración basal o de fondo del 


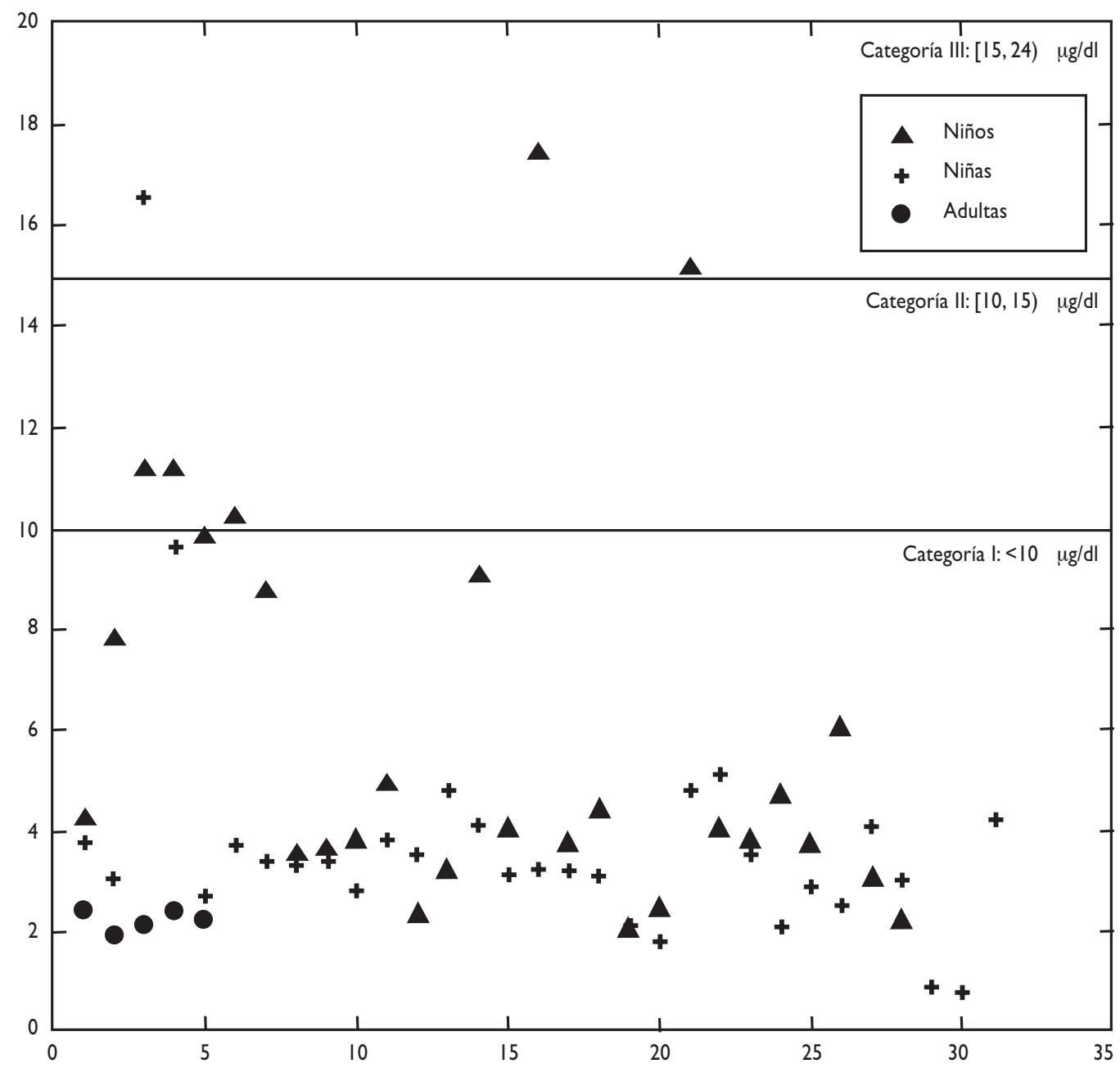

Figura 3. Concentración de Pb en sangre en niñas, niños y adultas de la comunidad de San Ignacio, Fresnillo, Zacatecas, separados por las categorías definidas por la Norma Oficial Mexicana." El muestreo se REALIZÓ DURANTE EL MES DE MARZO DE 2005

plomo. La muestra 26 se obtuvo de un punto alejado de la planta que se ubica fuera de la dirección preferencial del viento, que va de sur a norte. La concentración de plomo en el suelo de San Ignacio varía de 65 a $205 \mu \mathrm{g} / \mathrm{g}$, y el valor promedio es $109 \pm 43 \mu \mathrm{g} / \mathrm{g}$.

$\mathrm{La}$ concentración de $\mathrm{Pb}$ en las cenizas recuperadas de las casas de las tres familias es 311, 152 y 159 $\mu \mathrm{g} / \mathrm{g}$, el suelo del interior de sus viviendas tiene 205, 174 y $112 \mu \mathrm{g} / \mathrm{g}$, mientras que en la parte externa hay 74,80 y $80 \mu \mathrm{g} / \mathrm{g}$, respectivamente. De estos valores se observa que las cenizas y el suelo del interior de las viviendas tienen una mayor concentración de plomo que supera al valor basal.

Entre la parte externa e interna de la olla de barro existe una diferencia en la concentración de $\mathrm{Pb}$ de
$30443 \pm 1808 \mu \mathrm{g} / \mathrm{g}$. Si se parte de suponer que la olla se utilizó a diario en los últimos 10 años, se infiere que el plomo se transfirió durante el cocimiento de los frijoles a una razón de $3044 \mu \mathrm{g} / \mathrm{g} / \mathrm{año}$, lo que arroja una tasa de transferencia de $83 \mu \mathrm{g} / \mathrm{g} /$ día. Para probar lo anterior, se midió el plomo en frijoles crudos y cocidos en la olla. Los frijoles crudos tienen una concentración de plomo de $87 \mu \mathrm{g} / \mathrm{g}$, mientras que los cocidos tienen $175 \mu \mathrm{g} / \mathrm{g}$. La diferencia, $88 \mu \mathrm{g} / \mathrm{g}$, coincide con la tasa de transferencia calculada e indica que una parte del plomo de la olla se moviliza hacia el alimento durante la cocción.

De los terrenos próximos a la recicladora se obtuvieron muestras de nopal tapón (Opuntia robusta), pachón (Opuntia spp) y duraznillo (Opuntia leucotricha). Las concentraciones de plomo en los nopales son 
Cuadro I

Valores promedio de la concentración de Pb EN SANGRE DE MENORES, EMBARAZADAS Y MUJERES EN PERIODO DE LACTANCIA POR GRUPO DE EDAD, género y en SU CONJUnto, en San Ignacio, Fresnillo, Zacatecas (el muestreo se realizó DURANTE EL MES DE MARZO DE 2005)

\begin{tabular}{lccc}
$\begin{array}{c}\text { Grupo de edad } \\
\text { [años] }\end{array}$ & $\begin{array}{c}\text { Varones } \\
{[\mu \mathrm{g} / \mathrm{dl}]}\end{array}$ & $\begin{array}{c}\text { Mujeres } \\
{[\mu \mathrm{g} / \mathrm{dl}]}\end{array}$ & $\begin{array}{c}\text { Mujeres y varones } \\
{[\mu \mathrm{g} / \mathrm{dl}]}\end{array}$ \\
$0-3$ & $6.9 \pm 3.4$ & $5.5 \pm 5.4$ & $6.0 \pm 4.6$ \\
\hline $3-6$ & $5.9 \pm 3.4$ & $3.4 \pm 1.1$ & $4.8 \pm 2.8$ \\
\hline $6-12$ & $6.5 \pm 4.7$ & $3.8 \pm 2.0$ & $5.3 \pm 3.9$ \\
\hline $12-15$ & $3.7 \pm 1.6$ & $2.8 \pm 1.3$ & $3.1 \pm 1.4$ \\
\hline Adultas & - & $2.2 \pm 0.2$ & -
\end{tabular}

$368 \mu \mathrm{g} / \mathrm{g}$ para el tapón, $440 \mu \mathrm{g} / \mathrm{g}$ para el duraznillo y $1952 \mu \mathrm{g} / \mathrm{g}$ para el pachón, mientras que el camote tiene $422 \mu \mathrm{g} / \mathrm{g}$ del metal. La concentración de $\mathrm{Pb}$ en el chile, obtenido de los terrenos próximos a la recicladora, es de $158 \mu \mathrm{g} / \mathrm{g}$, mientras que el usado como testigo tiene $87 \mu \mathrm{g} / \mathrm{g}$.

\section{Discusión}

Todas las adultas analizadas tienen valores de plomo en sangre inferiores a los $10 \mathrm{mg} / \mathrm{dl}$. De los 59 niños analizados, 90\% muestra cifras inferiores a los $10 \mu \mathrm{g} / \mathrm{dl}$, mientras que $10 \%$ exhibe concentraciones superiores a $\operatorname{los} 10 \mu \mathrm{g} / \mathrm{dl}$. De este $10 \%$, el $50 \%$ presenta valores entre 10 y $15 \mu \mathrm{g} / \mathrm{dl}$ y el otro $50 \%$, entre 15 y $18 \mu \mathrm{g} / \mathrm{dl}$. En promedio, todos los menores tienen una concentración de $\mathrm{Pb}$ en sangre de $4.9 \mu \mathrm{g} / \mathrm{dl}$, que es igual al de los niños de la zona metropolitana ${ }^{15}$ después de la eliminación de la gasolina con plomo. El hecho de que niños del área rural así como de zonas urbanas presenten concentraciones promedio similares permite establecer la existencia de un nivel basal, aunque es necesario realizar este tipo de estudios en grupos de niños de otras áreas del país.

La concentración de $\mathrm{Pb}$ en la sangre de los niños es mayor al que se verifica en las adultas, $2.2 \mu \mathrm{g} / \mathrm{dl}$, debido a la diferencia en las características metabólicas, las actividades físicas y los hábitos higiénicos entre niños y adultos. En los grupos de edad de 0 a 3,3 a 6,6 a 12 y de 12 a 15 años no se encontró una diferencia estadísticamente significativa $(p=0.05)$ entre los valores de plomo en sangre encontrado en niños y niñas; no obstante, en conjunto, el valor promedio de $\mathrm{Pb}$ en sangre en las niñas presentó una diferencia estadísticamente significativa de acuerdo con la prueba de $\mathrm{U}$ de Mann y Whitney. ${ }^{12}$

salud pública de méxico / vol.48, no.3, mayo-junio de 2006
Cuadro II

Porcentaje de menores cuya concentración de Pb en sangre supera los 10 mg/dL, en San Ignacio, Fresnillo, Zacatecas (el muestreo SE REALIZÓ DURANTE EL MES DE MARZO DE 2005)

\begin{tabular}{lccc}
$\begin{array}{l}\text { Grupo de edad } \\
\text { [años] }\end{array}$ & $\begin{array}{c}\text { Niños } \\
{[\%]}\end{array}$ & $\begin{array}{c}\text { Niñas } \\
{[\%]}\end{array}$ & $\begin{array}{c}\text { Niñas y niños } \\
{[\%]}\end{array}$ \\
$0-3$ & 0 & 17 & 11 \\
\hline $3-6$ & 20 & 0 & 11 \\
\hline $6-12$ & 25 & 0 & 14 \\
\hline $12-15$ & 0 & 0 & 0 \\
\hline
\end{tabular}

La probable explicación de esta diferencia se atribuye a que los niños realizan una mayor actividad física en contacto con el suelo que las niñas y que, en términos generales, las niñas observan mejores hábitos higiénicos; esto coincide con lo que Jiménez-Gutiérrez y colaboradores informaron. ${ }^{16}$ En el cuadro II se constata que ningún menor entre los 12 y los 15 años de edad exhibe más de $10 \mu \mathrm{g} / \mathrm{dl}$, y la posible explicación del hecho consiste en que, a pesar de que sus miembros han de entrar en contacto con fuentes de plomo, su actividad y hábitos higiénico-dietéticos los hace menos susceptibles a incorporar este metal en su organismo.

Los niños cuyas edades varían de 3 a 12 años constituyen el grupo de mayor riesgo; en este caso la causa de su contaminación se atribuye a la actividad física y los deficientes hábitos de higiene propios de la edad.

En el caso de las niñas, el grupo de mayor riesgo tiene edades inferiores a los tres años. La situación de este grupo se debe a que sus integrantes interactúan de manera permanente con entidades con plomo, como el polvo del ambiente de sus casas y el suelo. ${ }^{17}$ Además, por su corta edad, los individuos de este grupo presentan un hábito marcado de llevarse objetos a la boca, ${ }^{2,3,7,8}$ sin que importe el grado de higiene de los mismos.

La mayoría de los niños de esta población presenta concentraciones de $\mathrm{Pb}$ en sangre por debajo de los $10 \mu \mathrm{g} / \mathrm{dl}$; para la NOM, esta concentración ubica al afectado en el nivel I y no se establece ninguna acción específica para su protección. Sin embargo, algunos autores ${ }^{1,18,19}$ han señalado que se producen daños a la salud de los niños aun a concentraciones bajas de plomo. Si bien es cierto que se han abatido los valores de plomo en sangre, la exposición infantil al metal se mantiene como un problema de salud pública, de donde resulta necesario continuar con los trabajos de investigación que conduzcan a identificar las zo- 


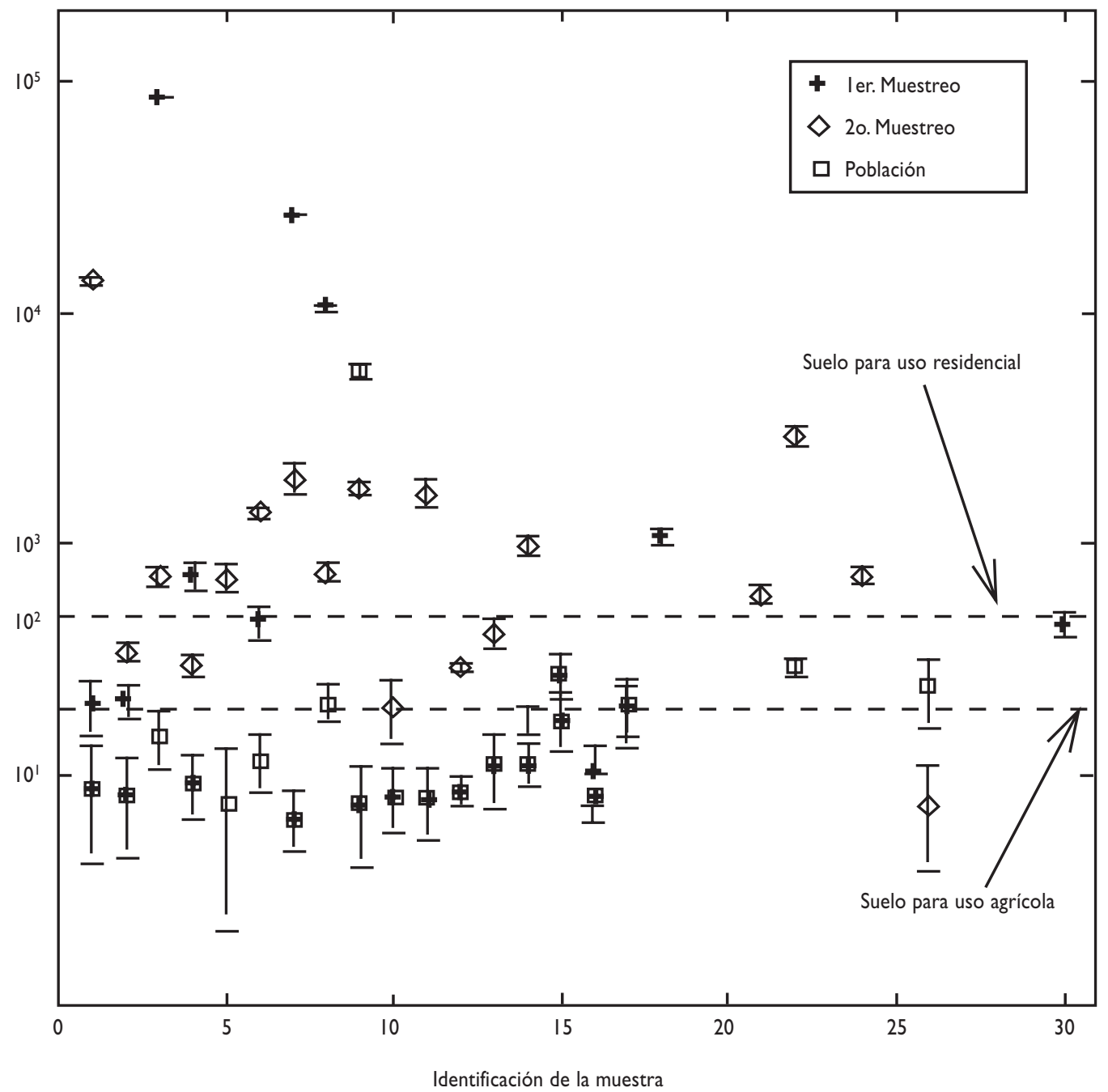

Figura 4. Concentración de Pb en suelo de los terrenos, de agostadero y cultivo, localizados en torno a la recicladora, así como del poblado de San Ignacio, Fresnillo Zacatecas. Se muestran dos líneas que indican LOS LÍMITES ESTABLECIDOS PARA EL USO DEL SUELO. LAS MUESTRAS SE TOMARON EN LOS MESES DE ENERO Y ABRIL DE 2005

nas de alto riesgo y determinar las concentraciones de $\mathrm{Pb}$ en sangre de la población más vulnerable.

El valor promedio de la concentración de $\mathrm{Pb}$ en el suelo de los terrenos aledaños a la recicladora supera los $500 \mu \mathrm{g} / \mathrm{g}$ que señala la legislación canadiense para uso residencial y los $200 \mu \mathrm{g} / \mathrm{g}$ señalados como límite para uso agrícola. ${ }^{20}$ Del análisis de las figuras 2 y 4 se puede concluir que la concentración de plomo en suelo varía en proporción inversa a la distancia de la recicladora, situación similar a la que se verifica en la ciudad de Torreón. ${ }^{20}$ La concentración promedio del suelo de San Ignacio coincide con lo comunicado en San Luis Potosi ${ }^{20}$ y representa la concentración de fondo o basal de $\mathrm{Pb}$ en suelo. Este valor confirma que los terrenos aledaños a la recicladora están contaminados.
Este hecho también indica que el suelo de los terrenos contaminados no se transporta de manera importante al poblado, que se ubica aproximadamente a $90^{\circ}$ de la dirección preferencial del viento.

Las diferencias en el $\mathrm{Pb}$ encontrado en las cenizas y el suelo de las partes interior y exterior de las casas de las tres familias afectadas reflejan un proceso de enriquecimiento en la concentración de $\mathrm{Pb}$. El fenómeno tal vez responde a a la acumulación de partículas de ceniza y de polvo en el interior de las viviendas, donde la erosión eólica y pluvial es más limitada que la que ocurre en las calles.

El chile tiene 3.2\% del $\mathrm{Pb}$ encontrado en el valor promedio del suelo, y dicho valor coincide con el que informaron Finster y colaboradores. ${ }^{21}$ Este porcentaje 
es $8.5 \%$ para el caso del camote, $7.4 \%$ para el nopal tapón, $8.9 \%$ para el nopal duraznillo y $39.5 \%$ para el nopal pachón. La mayor parte de estas plantas es tolerante al $\mathrm{Pb}$, mientras que el nopal pachón es un hiperacumulador. Esta situación, junto con los pobres hábitos higiénicos, permite explicar la situación de 10\% de los niños cuya concentración de plomo los ubica en las categorías II y III.

Para el caso de la familia 3, otra fuente de plomo la constituye el uso de loza vidriada, que propicia que los miembros de esta familia ingieran plomo de forma regular. La cantidad de plomo que incorporan por esta vía supera el límite tolerable de $15 \mu \mathrm{g}$ de $\mathrm{Pb}$ por día que, para mayores de 7 años, señala la Administración de Alimentos y Medicamentos de Estados Unidos de América. ${ }^{21}$

El hecho de ubicar en medio de terrenos de uso agropecuario una empresa cuyo giro es el reciclado de metales es por demás irregular. Su actividad ha producido una contaminación de los terrenos de agostadero y cultivo que la rodean. La diferencia entre los valores de $\mathrm{Pb}$ en suelo en estos terrenos y los del poblado de San Ignacio son una prueba del pasivo ambiental de la empresa. Además, esta diferencia indica que los suelos contaminados no se transportan de manera notable a la comunidad. En los terrenos de cultivo afectados se producen alimentos que presentan concentraciones altas de plomo, cuyo consumo es una fuente de contaminación que afecta sobre todo a los menores de 15 años, ${ }^{1,18,21}$ situación que se agrava si se utiliza barro vidriado para la preparación de los alimentos. El otro grupo de terrenos afectados son para agostadero, donde bovinos y caprinos pastan, consumiendo el pienso contaminado. El consumo de los productos lácteos y la carne de estos animales también representa una fuente potencial de plomo.

\section{Agradecimientos}

Este proyecto recibió el apoyo parcial del Consejo Zacatecano de Ciencia y Tecnología (COZCyT) bajo el convenio FOMIX-ZAC-2003-C01-0107.

\section{Referencias}

I. Koller K, Brown T, Spurgeon A, Levy L. Recent developments in lowlevel lead exposure and intellectual impairment in children. Environ Health Persp 2004; I 12:987-994.

2. Mielke HW, Dugas D, Mielke PW, Smith KS, Smith SL, Gonzales ChR. Associations between soil lead and childhood blood lead in urban New Orleans and rural Lafourche Parish of Louisiana. Environ Health Persp 1997; 105:950-954.
3. Mielke HW, Reagan PL. Soil is an important pathway of human lead exposure. Environ Health Persp 1998; 106:217-229.

4. Lanphear BP, Howard C, Eberly S, Auinger P, Kolassa J,Weitzman M et al. Primary prevention of childhood lead exposure: a randomized trial of dust control. Pediatrics 1999;103:772-777.

5. Lawendon G, Kinra S, Nelder R, Cronin T. Should children with developmental and behavioral problems be routinely screened for lead?. Arch Dis Childhood 200I;85:286-288.

6. Canfield RL, Gendle MH, Cory-Slechta DA. Impaired neuropsychological functioning in lead-exposed children. Develop Neuropsy 2004;26:5।3-540.

7. Mielke HW, Gonzales CR, Smith MK, Mielke PW. The urban environment and children's health: soils as integrator of lead, zinc, and cadmium in New Orleans, Louisiana, USA. Environ Health Persp 1999;81:1 17-129.

8. Rahbar MH,White F, Agboatwalla M, Hozhabri S, Luby S. Factors associated with elevated blood lead concentrations in children in Karachi, Pakistan. Bull World Health Organ 2002;80:769-775.

9. Lowry LK, Cherry DC, Brady ChF, Huggins B, D'Sa AM, Levin JL. An unexplained case of elevated blood lead in hispanic child. Environ Health Persp 2004; I 2:222-225.

10. Manzanares-Acuña E, Vega-Carrillo HR, Letechipía-de León C, Guzmán-Enríquez LJ, Hernández-Dávila VM, Salas-Luévano MA. Estudio del perfil elemental en suelo de $\mathrm{Hg}$ y $\mathrm{Pb}$ en sangre en niños menores de 5 años $y$ en embarazadas en Vetagrande, Zacatecas. Reporte de investigación UaEN/RI-02-PbHgVG/INE-02/0 I 24I I04. Zacatecas, México: Universidad Autónoma de Zacatecas, 2004.

I I. Norma Oficial Mexicana NOM-I99-SSAI-2000. Salud ambiental. Niveles de plomo en sangre y acciones como criterios para proteger la salud de la población expuesta no ocupacionalmente. Diario Oficial de la Federación del viernes 18 de octubre de 2002. México, D.F.: Secretaría de Salud, 2002.

12. Lyman-Ott R. An introduction to statistical methods and data analysis. Fourth edition. Belmont: Duxbury Press, 1993:279-284. 13. Manzanares-Acuña E,Vega-Carrillo HR, Letechipía-de León C, SalasLuévano MA, Hernández-Dávila VM. Estudio sobre la concentración de plomo en muestras de suelo y plantas obtenidas en torno a la empresa "Gildardo Gómez y/o Reciclado de metales". Reporte de investigación UaEN/RI-0I-PbSI/07/UAZ-0I/05101204. Zacatecas, México: Universidad Autónoma de Zacatecas, 2005.

14. Vega-Carrillo HR. Least squares for different experimental cases. Revista Mexicana de Física 1989;35:597-602.

15. Palazuelos-Rendón E. Plomo y salud. Impacto ambiental de la reformulación de las gasolinas en la zona metropolitana de la Ciudad de México. Una evaluación económica. En: Hernández-Ávila M, PalazuelosRendón E, ed. Intoxicación por plomo en México: Prevención y control. México. Cuernavaca, México: Instituto Nacional de Salud Pública, 1995;257-288.

16. Jiménez-Gutiérrez C, Romieu I, Ramírez-Sánchez AL, PalazuelosRendón E, Muñóz-Quiles I. Exposición a plomo en niños de 6 a 12 años de edad. Salud Publica Mex 1999;41:s72-s8I.

17. Romieu I, Palazuelos E, Hernández-Ávila M, Ríos C, Muñoz I, Jiménez $C$ et al. Sources of lead exposure in Mexico City. Environ Health Persp 1994;102:384-389.

18. Meneses-González F, Richardson V, Lino-González M,Vidal MT. Niveles de plomo en sangre y factores de exposición en niños del estado de Morelos, México. Salud Pública Mex 2003; 45: s203-s208. 19. Matte TD. Efectos del plomo en la salud de la niñez. Salud Publica Mex 2003;45:s220-s224.

20. Flores J,Albert LA. Environmental lead in Mexico, 1999-2002. Rev Environ Contam Toxicol 2004;181:37-100.

21 . Finster ME, Gray KA, Binns HJ. Lead levels of edible grown in contaminated residencial soils: A field survey. Sci Total Environ 2004;320:245-257. 